\title{
Front Matter: Volume 9435
}

, "Front Matter: Volume 9435," Proc. SPIE 9435, Sensors and Smart Structures Technologies for Civil, Mechanical, and Aerospace Systems 2015, 943501 (11 May 2015); doi: 10.1117/12.2193850

Event: SPIE Smart Structures and Materials + Nondestructive Evaluation and SPIE. Health Monitoring, 2015, San Diego, California, United States 


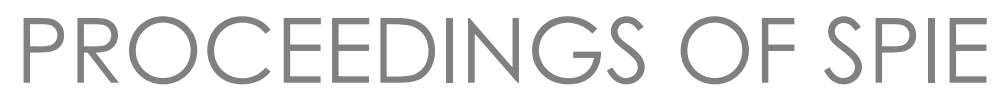

\title{
Sensors and Smart Structures Technologies for Civil, Mechanical, and Aerospace Systems 2015
}

\author{
Jerome P. Lynch \\ Kon-Well Wang \\ Hoon Sohn \\ Editors
}

9-12 March 2015

San Diego, California, United States

Sponsored by

SPIE

Cosponsored by

American Society of Mechanical Engineers (United States)

Cooperating Organizations

Intelligent Materials Forum (Japan)

Jet Propulsion Laboratory (United States)

National Science Foundation (United States)

Published by

SPIE

Volume 9435 
The papers included in this volume were part of the technical conference cited on the cover and title page. Papers were selected and subject to review by the editors and conference program committee. Some conference presentations may not be available for publication. The papers published in these proceedings reflect the work and thoughts of the authors and are published herein as submitted. The publisher is not responsible for the validity of the information or for any outcomes resulting from reliance thereon.

Please use the following format to cite material from this book:

Author(s), "Title of Paper," in Sensors and Smart Structures Technologies for Civil, Mechanical, and Aerospace Systems 2015, edited by Jerome P. Lynch, Kon-Well Wang, Hoon Sohn, Proceedings of SPIE Vol. 9435 (SPIE, Bellingham, WA, 2015) Article CID Number.

ISSN: 0277-786X

ISBN: 9781628415384

Published by

SPIE

P.O. Box 10, Bellingham, Washington 98227-0010 USA

Telephone +1 3606763290 (Pacific Time) · Fax +1 3606471445

SPIE.org

Copyright @ 2015, Society of Photo-Optical Instrumentation Engineers.

Copying of material in this book for internal or personal use, or for the internal or personal use of specific clients, beyond the fair use provisions granted by the U.S. Copyright Law is authorized by SPIE subject to payment of copying fees. The Transactional Reporting Service base fee for this volume is $\$ 18.00$ per article (or portion thereof), which should be paid directly to the Copyright Clearance Center (CCC), 222 Rosewood Drive, Danvers, MA 01923. Payment may also be made electronically through CCC Online at copyright.com. Other copying for republication, resale, advertising or promotion, or any form of systematic or multiple reproduction of any material in this book is prohibited except with permission in writing from the publisher. The CCC fee code is 0277-786X/15/\$18.00.

Printed in the United States of America.

Publication of record for individual papers is online in the SPIE Digital Library.

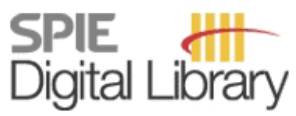

SPIEDigitalLibrary.org

Paper Numbering: Proceedings of SPIE follow an e-First publication model, with papers published first online and then in print. Papers are published as they are submitted and meet publication criteria. A unique citation identifier (CID) number is assigned to each article at the time of the first publication. Utilization of CIDs allows articles to be fully citable as soon as they are published online, and connects the same identifier to all online, print, and electronic versions of the publication. SPIE uses a six-digit CID article numbering system in which:

- The first four digits correspond to the SPIE volume number.

- The last two digits indicate publication order within the volume using a Base 36 numbering

system employing both numerals and letters. These two-number sets start with 00, 01, 02, 03, 04,

05, 06, 07, 08, 09, 0A, OB ... 0Z, followed by 10-1Z, 20-2Z, etc.

The CID Number appears on each page of the manuscript. The complete citation is used on the first page, and an abbreviated version on subsequent pages. 


\title{
Contents
}

\section{Part One}

\author{
xi Authors \\ xv Conference Committee
}

\section{KEYNOTE SESSION}

943502 New trends in non-destructive assessment of aerospace structures (Keynote Paper) [9435-1]

\section{SESSION 1 NOVEL APPROACHES TO ENERGY HARVESTING}

943504 New insights into vibration-based energy harvesting [9435-3]

943505 A frequency study of a clamped-clamped pipe immersed in a viscous fluid conveying internal steady flow for use in energy harvester development as applied to hydrocarbon production wells [9435-4]

943506 Design, modeling and test of a novel speed bump energy harvester [9435-5]

$943507 \quad$ Fluid flow nozzle energy harvesters [9435-6]

943508 Energy harvesting of two cantilever beams structure: interfacing circuit discussion [9435-7]

\section{SESSION 2 VIBRATIONS AND SHM OF BRIDGE STRUCTURES}

943509 Mitigating the effects of variable speed on drive-by infrastructure monitoring [9435-8]

$94350 \mathrm{~A}$ Intelligent tires for identifying coefficient of friction of tire/road contact surfaces [9435-9]

$9435 \mathrm{OB} \quad$ Recovering bridge deflections from collocated acceleration and strain measurements [9435-10]

9435 OC Analysis of traffic-induced vibration and damage detection by blind source separation with application to bridge monitoring [9435-1 1] 
9435 OE Development of a portable electrical impedance tomography data acquisition system for near-real-time spatial sensing [9435-13]

9435 OF Structural damage detection via impediographic tomography [9435-130]

\section{SESSION $4 \quad$ NANOENGINEERED THIN FILM SENSORS I}

9435 OG Sensing sheets based on large area electronics for fatigue crack detection [9435-15]

94350 Ol Peridynamics as an analysis tool for wave propagation in graphene nanoribbons [9435-17]

9435 0J Design and fabrication of mechanochromic photonic crystals as strain sensor [9435-18]

\section{SESSION 5 ADVANCED ULTRASONIC METHODS FOR SHM I}

9435 OK Design of embedded acoustic lenses in plate-like structures based on periodic acoustic black holes [9435-19]

$9435 \mathrm{OL} \quad$ Granular chains for the assessment of thermal stress in slender structures [9435-20]

9435 OM Frequency wavenumber design of spiral macro fiber composite directional transducers (Best Student Paper Award) [9435-21]

9435 ON Modulation scheme of nonlinear waves for effective crack detection [9435-22]

943500 Thermal effect on E/M impedance spectroscopy of piezoelectric wafer active sensors [9435-23]

\section{SESSION 6 NOVEL SYSTEM IDENTIFICATION AND DAMAGE DETECTION STRATEGIES}

9435 OP A data management infrastructure for bridge monitoring [9435-24]

$94350 Q \quad$ Uncertainty calculation for modal parameters used with stochastic subspace identification: an application to a bridge structure [9435-25]

9435 OR Substructure location and size effects on decentralized model updating [9435-26]

9435 OS Sequential damage detection based on the continuous wavelet transform [9435-27]

9435 OT Stochastic filtering for damage identification through nonlinear structural finite element model updating [9435-28]

9435 OU Sampling considerations for modal analysis with damping [9435-29] 
SESSION 7A BIG DATA AND DATA ANALYTICS FOR SHM

9435 OW Data management for biofied building [9435-31]

SESSION 7B SENSING SYSTEMS FOR FLIGHT CONTROL

9435 OX Gust alleviation of highly flexible UAVs with artificial hair sensors [9435-32]

9435 OY Low frequency motion measurement and control of spacecrafts and satellites [9435-33]

SESSION 8A ACTUATION AND FEEDBACK CONTROL TECHNOLOGIES

$94350 Z$ Sparse feedback structures for wireless control of civil systems [9435-34]

943510 A driven active mass damper by using output of a neural oscillator (effects of position control system changes on vibration mitigation performance) [9435-35]

943511 Low-force magneto-rheological damper design for small-scale structural control experimentation [9435-36]

943512 Development and application of a vibration isolation system with adaptive stiffness considering potential energy [9435-37]

\section{SESSION 8B NONLINEAR ULTRASONICS FOR DAMAGE DETECTION}

943513 Detection of fatigue crack on a rotating steel shaft using air-coupled nonlinear ultrasonic modulation [9435-38]

943514 Volumetric loss quantification using ultrasonic inductively coupled transducers [9435-39]

943516 Optimal design of force magnification frame of a piezoelectric stack energy harvester [9435-93]

\section{SESSION 9A SHM OF CONCRETE STRUCTURES}

943519 Development of a relationship between external measurements and reinforcement stress [9435-43]

$94351 \mathrm{~A}$ Multifractal analysis of two-dimensional images for damage assessment of reinforced concrete structures [9435-44]

9435 1B Experiment on interface separation detection of concrete-filled steel tubular arch bridge using accelerometer array [9435-45] 
9435 1D Development of Arduino based wireless control system [9435-47]

9435 1E A magnetorheological elastomer compressive and shear sensor [9435-48]

9435 IF Wireless ultrasound pitch-catch sensor powered by microwave energy [9435-49]

9435 IG Packet loss and compensation of Wi-Fi-based wireless sensor networks [9435-50]

$94351 \mathrm{H} \quad$ Cable force monitoring system of cable stayed bridges using accelerometers inside mobile smart phone [9435-51]

SESSION 10A NANOENGINEERED THIN FILM SENSORS II

$94351 \mathrm{~K}$ A remote-readable graphite oxide (GO) based tamper-evident seal with self-reporting and self-authentication capabilities [9435-54]

$94351 \mathrm{~N} \quad$ Algorithm for decomposition of additive strain from dense network of thin film sensors [9435-57]

\section{Part Two}

\section{SESSION 1OB ADVANCED ULTRASONIC METHODS FOR SHM II}

943510 Non-contact ultrasonic guided wave inspection of rails: field test results and updates [9435-58]

$94351 \mathrm{P} \quad$ Impact induced delamination detection and quantification with guided wavefield analysis [9435-59]

9435 IR Structural damage identification using piezoelectric impedance and Bayesian inference [9435-61]

9435 is Spira Mirabilis: a shaped piezoelectric sensor for impact localization [9435-62]

$94351 \mathrm{~T}$ Influence of higher and sub harmonics in time reversed Lamb wave generated and sensed using de-bonded piezoelectric wafers [9435-63]

SESSION 11A HUMAN AND HUMANOID SENSING AND ACTUATION

$94351 \mathrm{U} \quad$ Recognition of human activities using depth images of Kinect for biofied building [9435-64]

$94351 \mathrm{~V}$ The smart Peano fluidic muscle: a low profile flexible orthosis actuator that feels pain [9435-65] 
$9435 \mathrm{IW}$ A wind turbine hybrid simulation framework considering aeroelastic effects [9435-66]

9435 1X Adaptive pitch control for load mitigation of wind turbines [9435-67]

SESSION 12A FIBER OPTIC SENSING TECHNOLOGY

9435 IY Seven-year-long crack detection monitoring by Brillouin-based fiber optic strain sensor [9435-68]

$94351 \mathrm{Z}$ Pipeline corrosion assessment using embedded Fiber Bragg grating sensors [9435-69]

943520 Damage monitoring of CFRP retrofit using optical fiber sensors [9435-70]

$943521 \quad$ Evaluation of the use of fiber optic sensors in identification of fresco fracturing patterns [9435-71]

\section{SESSION 12B VISION-BASED SENSING TECHNOLOGIES}

943522 Hyperspectral imaging utility for transportation systems [9435-72]

943523 A digital sampling moiré method for two-dimensional displacement measurement [9435-73]

943524 Strain characterization of embedded aerospace smart materials using shearography [9435-74]

943525 Stereovision-based method for the whole process of collapse measurement of a threefloor frame model under earthquake excitation [9435-75]

\section{SESSION 13A APPLICATIONS OF SMART STRUCTURE TECHNOLOGIES}

943527 Thermal analysis of brazing seal and sterilizing technique to break contamination chain for Mars sample return [9435-77]

943528 Spiral passive electromagnetic sensor (SPES) for smart sensing and de-icing [9435-78]

943529 Heat transfer computed tomography techniques for damage detection in metallic structures [9435-14]

9435 2A Testing of tactile sensors for space applications [9435-79]

\section{SESSION 13B NEXT-GENERATION SENSORS FOR SMART STRUCTURES}

9435 2B The design, characterization, and comparison of MEMS comb-drive acoustic emission transducers with the principles of area-change and gap-change [9435-80] 
9435 2D A coin size, 40mW, 20 grams sensor node for guided waves detection [9435-82]

$94352 \mathrm{E}$ Quantification of seismic damage in steel beam-column connection using PVDF strain sensors and model-updating technique [9435-83]

SESSION 14A ADVANCED COMPOSITE STRUCTURAL SYSTEMS

$94352 \mathrm{G}$ Relation between repeatability and speed of robot-based systems for composite aircraft production through multilateration sensor system [9435-85]

$943521 \quad$ Self sensing composites with emi shielding and self repair [9435-88]

$94352 \mathrm{~J} \quad$ Modelling of shear lag effect for piezo-elstodynamic structure for electro-mechanical imedance technique [9435-89]

\section{SESSION 14B MODELING TECHNIQUES FOR SMART STRUCTURES}

$94352 \mathrm{~K} \quad$ Structural damage detection using extended Kalman filter combined with statistical process control [9435-90]

$94352 \mathrm{~L} \quad$ Variation of modal properties by combined effects of temperature and boundary condition [9435-91]

$94352 \mathrm{M}$ On predicting monitoring system effectiveness [9435-92]

POSTER SESSION

$94352 Q \quad$ Low frequency seismic characterization of underground sites with funable mechanical monolithic sensors [9435-97]

$94352 R \quad$ Nap environment control considering respiration rate and music tempo by using sensor agent robot [9435-100]

$94352 \mathrm{~S}$ Homeostasis lighting control based on relationship between lighting environment and human behavior [9435-101]

9435 2T Software design and implementation of ship heave motion monitoring system based on MBD method [9435-102]

$94352 \mathrm{U}$ Statistical analysis of nature frequencies of hemispherical resonator gyroscope based on probability theory [9435-103]

$94352 \mathrm{~V}$ Inductive wireless sensor-actuator node for structural health monitoring of fiber reinforced polymers by means of Lamb-waves [9435-104]

9435 2W In-situ strain sensing with fiber optic sensors embedded into stainless steel 316 [9435-105] 
$94352 X \quad$ Innovative insulations for spacecraft on-surface monitoring system in harsh environments [9435-106]

$94352 Y \quad$ Liquid polyimide as a substrate for aeronautical sensor systems [9435-107]

943530 Research on slope instability displacement monitoring technique based on laser spot video identification method [9435-109]

943531 Emotion identification method using RGB information of human face [9435-110]

943533 Simultaneous shear and pressure sensing based on patch antenna [9435-1 12]

943535 Influence of surface processing on the fracture strength of structurally integrated PZT fibers in shaped sheet metal parts [9435-114]

943536 Dynamic pressure sensor calibration techniques offering expanded bandwidth with increased resolution [9435-115]

943537 Pressure sensing using low-cost microstrip antenna sensor [9435-116]

943538 Damage identification via asymmetric active magnetic bearing acceleration feedback control [9435-117]

943539 An investigation of the material and model parameters for a constitutive model for MSMAs [9435-118]

$94353 \mathrm{~A}$ Structural damage detection for in-service highway bridge under operational and environmental variability [9435-119]

9435 3D Flexible patch composed of PZT thin-film on stainless steel foil for energy harvesting from low-frequency human motions [9435-122]

$94353 \mathrm{E}$ Identification of sensor fault for the performance and stability of centralized control logics [9435-123]

$94353 \mathrm{~F} \quad$ Fault detection in small diameter pipes using ultrasonic guided wave technology [9435-124]

9435 3G Design and simulation of multi-resonance sonic transducer using Terfenol-D [9435-125]

$94353 \mathrm{H} \quad$ Comparative discussion between first and second modes of Terfenol-D transducer [9435-126]

943531 Characterization of a soft elastomeric capacitive strain sensor for fatigue crack monitoring [9435-127]

9435 3K Regenerative magnetorheological dampers for vehicle suspensions [9435-129] 


\section{Authors}

Numbers in the index correspond to the last two digits of the six-digit citation identifier (CID) article numbering system used in Proceedings of SPIE. The first four digits reflect the volume number. Base 36 numbering is employed for the last two digits and indicates the order of articles within the volume. Numbers start with 00, 01, 02, 03, 04, 05, 06, 07, 08, 09, 0A, 0B...0Z, followed by 10-1Z, 20-2Z, etc.

\author{
Acernese, F., OY, 2Q \\ Anderson, lain A., 1V \\ Anisimov, Andrei G., 24 \\ Astroza, Rodrigo, OT \\ Azarmi, Fardad, 1Z, 2X \\ Badescu, Mircea, 27 \\ Bae, In Hwan, OP \\ Bagheri, Abdollah, OL \\ Balafas, Konstantinos, OS \\ Bao, Xiaoqi, 27 \\ Bao, Yuequan, $1 \mathrm{G}$ \\ Bar-Cohen, Yoseph, 27 \\ Barone, F., OY, 2Q \\ Bell, M., OB \\ Bennett, Caroline, 3l \\ Beykirch, B., 2G \\ Bhalla, Suresh, 2J \\ Bielak, Jacobo, 09 \\ Bijudas, C. R., $1 T$ \\ Bock, M., 2G \\ Bossert, Jason A., $1 \mathrm{~K}$ \\ Breaux Frketic, Jolie, 20 \\ Brault, Andre, 19 \\ Bridgelall, Raj, 22 \\ Burton, Andrew, 2E \\ Büttgenbach, Stephanus, $2 Y$ \\ Byun, Jaewook, OP \\ Cappello, Carlo, 2M \\ Carrara, Matteo, OM \\ Cattaneo, A., $1 \mathrm{~K}$ \\ Cazzulani, Gabriele, 3E \\ Chang, Chih-Chen, 23 \\ Chao, Shu-Hsien, $\mathrm{OQ}$ \\ Chen, Chao Ting, 3D \\ Chen, Chao, 3K \\ Chen, Chi-Jen, 12 \\ Chen, Jiun Jung, 3D \\ Chen, Sheng-Fu, OC \\ Chen, Shubin, 16 \\ Chen, Xinxing, 23 \\ Chen, Yu-Yin, 08 \\ Chernow, Barbara, 06 \\ Chiappini, A., OJ \\ Christenson, Richard, 3A \\ Cinquemani, Simone, 3E, 3G, 3H \\ Ciocanel, Constantin, 39 \\ Colonius, Tim, 07 \\ Conte, Joel P., OT \\ De Marchi, L., 1S, 2D
}

De Rosa, R., 2Q

DeSmidt, Hans, 38

DeVallance, David B., 2A

Dickens, Tarik, 20

Dietzel, Andreas, $2 Y$

Dikes, Jason, 39

Dobkin, David P., 21

Dong, Xinjun, OR

Downey, Austin, $1 \mathrm{~N}$

Dry, Carolyn, 21

Dumont, Joseph H., $1 \mathrm{~K}$

Dyke, Shirley J., 1D

Ebrahimian, Hamed, OT

Ebrahimkhanlou, Arvin, 1A

Evans, Thomas, 2A

Farhidzadeh, Alireza, 1A

Farrar, Charles R., 1K

Fateh, Mahmood, 10

Feigenbaum, Heidi, 39

Ferrari, M., OJ

Ferraro, A., 2D

Focke, Oliver, 2V

Frankforter, Erik, 00

Galedari, Sahar Abuali, 12

Ghafoorianfar, Nima, 1E

Ghodsi, Mojtaba, 3G, 3H

Gilbert, Anna C., OU

Giordano, G., OY, 2Q

Giurgiutiu, Victor, 00

Glisic, Branko, 0G, 21, 2M

Gong, Peng, 14

Gordaninejad, Faramarz, 1E

Greve, David W., 14

Groves, Roger M., 24

Gupta, Gautam, 1K

Guzman, Christian, 1K

Haaker, Axel, IK

Han, Feng, $1 G$

Han, Ruicong, $1 \mathrm{H}$

Hand, Duncan P., 2W

Harrigan, Terence, 06

Havermann, Dirk, 2W

Hay, Thomas R., 14

Hecht, Lars, $2 Y$

Herrmann, Axel S., 2V

Hojjat, Yousef, 3G, 3H

Hong, Jung-Wuk, ON

Hongu, J., 10

Hoult, Neil A., 19 
Hsu, Wei-Ting, $0 Q$

$\mathrm{Hu}$, Weitong, $1 \mathrm{H}$

Huang, Haiying, 1F, 33, 37

Huang, Shieh-Kung, OE

Huang, Ying, 1Z, 2X

Humphrey, Victor, 3F

Hung, Tzu-Yun, OC

Huo, Xiaoyang, 25

lba, D., 10

lervolino, Onorio, 28

Imai, Michio, $1 Y$

Jaguan, M., 37

Jang, Shinae, 2K, 3A

Jazi, Mehdi Salimi, 2X

Jeffers, Ann E., 29

Jeong, Seongwoon, OP

Jiang, Hao, 33

Jiao, Dong, $1 \mathrm{H}$

Jin, Chenhao, 2K, 3A

Johnson, Nephi R., 29

Joshi, Kunal, 20

Kabir, Minoo, 2B

Kamai, Kazuto, OA

Kamas, Tuncay, 00

Kang, Won-Hee, 2T

Kim, Daeyoung, OP

Kiremidjian, Anne S., OS

Kita, Shinya, 31

Kjolsing, Eric, 05

Koch, Eugen Viktor, $2 Y$

Kogan, Lisa, 2A

Kong, Xiangxiong, 3I

Krombholz, C., 2G

Kurata, Masahiro, 2E

La Malfa Ribolla, Emma, OL

Laflamme, Simon, 1N, 31

Lang, Walter, $2 \mathrm{~V}$

Lanza di Scalea, Francesco, 10

Law, Kincho H., OP

Leckey, Cara A. C., IP

Lederman, George, 09

Lee, Hyeong Jae, 07

Lee, Sang Eon, ON

Lees, Janet M., 19

Leester-Schädel, Monika, $2 Y$

Li, Geng, $2 U$

Li, Jian, 3

Li, Jingcheng, 3A

$\mathrm{Li}$, Mingchu, $1 \mathrm{H}$

Li, Shuang, 25

Li, Xiaohua, 2E

Li, Yuhan, 2T

Liao, Wei-Hsin, 3K

Liao, Yizheng, OS

Liang, Xiao, $1 \mathrm{Z}$

Lim, Cheol-Woo, 13

Lin, Bin, 00

Lin, Tzu-Kang, 12

Linderman, Lauren E., $\mathrm{OZ}$

Liu, Hao, 30
Loh, Chin-Hsiung, OC, OQ

Loh, Kenneth J., OE

Long, Xingwu, $2 \mathrm{U}$

Lynch, Jerome P., 29

Ma, T. W., 04, OB

Macpherson, William N., 2W

Maier, Robert R. J., 2W

Malinowski, Pawel H, 02

Mao, Jian, 1B

Mao, Xingquan, $1 \mathrm{H}$

Marchi, Alexandria N., IK

Marciniak, M., OJ

Mariani, Stefano, 10

Martowicz, A., Ol

Marzani, A., 1S, 2D

Mascareñas, David D. L., 1K

Matamoros, Adolfo, 3l

Mathew, Jinesh, 2W

Matsuura, Kohta, OW

Matsuzaki, Ryosuke, OA

McAvoy, David, 06

Mears, A., 37

Meo, Michele, 28

Miller, Karen A., 1K

Minegishi, Kaede, 2E

Mita, Akira, OW, 1U, 2R, 2S, 31

Moharana, Sumedha, 2J

Mohite, Aditya, $1 \mathrm{~K}$

Moriwaki, I., 10

Moshrefi-Torbati, Mohamed, 3F

Müller, Bernhard, 24

Müller, Michael, 35

Musgrave, Patrick, 16

Nakamura, M., 10

Nakaso, Sayaka, 2R

Navaneetha Krishnan, J., IT

Nguyen, Thompson V., 10

Noh, Hae Young, 09

O'Connor, James, 06

Ogawa, Ami, $1 \mathrm{U}$

Olawale, David, 20

Oppenheim, Irving J., 14

Oshima, Yoshinobu, 09

Ostachowicz, Wieslaw M., 02

OU, Jinping, 1G, 1H, 2T

Ozevin, Didem, 2B

Pan, Shengshan, 1B

Pandey, Abhishek, 06

Park, Byeongjin, 13

Park, Jae Young, OU

Park, Jae-Roung, 13

Pena, Francisco, 1D

Perner, M., 2G

Piotrowska, A., OJ

Pozzi, Matteo, 2M

Purdy, Geraldine M., $1 \mathrm{~K}$

Qu, Tianliang, $2 U$

Rafert, J. Bruce, 22

Rajagopal, Ram, OS

Reich, Gregory W., OX 
Ripamonti, Francesco, 3E

Rizzo, Piervincenzo, OL

Romano, R., OY, $2 Q$

Ronchi, Marco, 3E

Ruzzene, Massimo, OI, OM

Sabhnani, Rahul M., 3F

Sabolsky, Edward M., 2A

Saboonchi, Hossain, 2B

Salamone, Salvatore, IA

Salas, Mariugenia, 2V

Saleem, Hussam, $1 \mathrm{~N}$

Sasaki, T., 10

Schmidt, Marek, 35

Schwerter, Martin, $2 Y$

Seebo, Jeffrey P., IP

Seki, Ryosuke, OA

Semperlotti, Fabio, OF, OK

Shan, Baohua, 25

Sherrit, Stewart, 07

Sheykholeslami, Mohammad Reza, 3G, 3H

Shuai, Q., 1R

Sigurdardottir, Dorotea, 21, 2M

Sinke, Jos, 24

Sohn, Hoon, OP, 13

Song, Byeongju, 13

Song, Wei, 1W, $2 \mathrm{~L}$

Staszewski, W. J., Ol

Su, Weihua, OX, IW

Sun, Xiaorong, 2K, 3A

Sun, Zhuoxiong, 1D

Suzuki, Akiko, 2E

Swartz, R. Andrew, 11

Tang, J., 1R, $1 X$

Tang, Zhenyun, 2E

Testoni, N., 1S, 2D

Thorsen, Andrew, 09

Tian, Zhenhua, $1 \mathrm{P}$

Tjuatja, S., 37

Todaria, Prakhar, 06

Todd, Michael, 05

Tolliver, Denver, 22

Tosi, Luis Phillipe, 07

Ueda, Risa, 25

Uhl, T., Ol

Vasic, Dejan, 08

Veale, Allan Joshua, IV

Velazquez, Antonio, 11

Verdoljak, Reuben D., OZ

Wakin, Michael B., OU

Walkemeyer, Phillip, 07

Wandowski, Tomasz, 02

Wang, K. W., OF

Wang, Lirong, 06, 16

Wang, Yang, OR

Wang, Yin Jie, 3D

Weadon, Timothy L., 2A

Wei, Guo, $2 U$

Wilbee, Alana, 1D

Winn, Tyler, 07

Winter, Benjamin D., 11
Wisniewiski, David, 36

Wittstock, Volker, 35

Wu, Wen Jong, 3D

Xie, Shane Q., IV

$X \cup, C . C ., 37$

$X \cup, N$. S., 04, OB

$X U$, Tian-Bing, 16

$\mathrm{Xu}, \mathrm{Xi}, 30$

Yang, Jie, OF

Yao, Jun, 1F, 37

Yao, Wei, 38

Yao, Yao, OG

Yeh, Sou Peng, 3D

Yu, Lingyu Lucy, 00, $1 P$

Yu, Xudong, $2 U$

Yu, Yan, 1G, 1H, 2T

Yuan, Wenting, 25

Yuan, Yuan, $1 \mathrm{X}$

Zaghari, Bahareh, 3F

Zahedi, Farshad, IF

Zhang, Chunwei, 2T

Zhang, H., 04

Zhao, Hailiang, 1B

Zhao, Jie, 38

Zhao, Liuxian, OF

Zhao, Xuefeng, 1B, 1H, 30

Zhou, K., IR

Zhou, Shanglian, $2 \mathrm{~L}$

Zhou, Wanlu, 16

Zhu, Dapeng, OR

Zhu, Hongfei, OK

Zhu, Xuan, 10

Zonta, Daniele, OJ, 2M

Zou, Li, 3K

Zuo, Lei, 06, 16 


\section{Conference Committee}

Symposium Chairs

Victor Giurgiutiu, University of South Carolina (United States)

Christopher S. Lynch, University of California, Los Angeles

(United States)

Symposium Co-chairs

Jayanth N. Kudva, NextGen Aeronautics, Inc. (United States)

Theodoros E. Matikas, University of loannina (Greece)

Conference Chair

Jerome P. Lynch, University of Michigan (United States)

Conference Co-chairs

Kon-Well Wang, University of Michigan (United States)

Hoon Sohn, KAIST (Korea, Republic of)

Conference Program Committee

Dumitru Caruntu, The University of Texas-Pan American (United States)

Fabio Casciati, Università degli Studi di Pavia (Italy)

Chih Chen Chang, Hong Kong University of Science and Technology

(Hong Kong, China)

Genda Chen, Missouri University of Science and Technology

(United States)

Alison B. Flatau, University of Maryland, College Park (United States)

Yozo Fujino, The University of Tokyo (Japan)

Branko Glisic, Princeton University (United States)

Faramarz Gordaninejad, University of Nevada, Reno (United States)

Xiaoyan Han, Wayne State University (United States)

Benjamin K. Henderson, Air Force Research Laboratory

(United States)

Jung-Wuk Hong, KAIST (Korea, Republic of)

Neil A. Hoult, Queen's University (Canada)

Haiying Huang, The University of Texas at Arlington (United States)

Ying Huang, North Dakota State University (United States)

Shinae Jang, University of Connecticut (United States)

Jeong-Tae Kim, Pukyong National University (Korea, Republic of)

Junhee Kim, Dankook University (Korea, Republic of)

Masahiro Kurata, Kyoto University (Japan) 
Simon Laflamme, lowa State University (United States)

Francesco Lanza di Scalea, University of California, San Diego

(United States)

Hui Li, Harbin Institute of Technology (China)

Wei-Hsin Liao, The Chinese University of Hong Kong

(Hong Kong, China)

Yingzi Lin, Northeastern University (United States)

Chin-Hsiung Loh, National Taiwan University (Taiwan)

Kenneth J. Loh, University of California, Davis (United States)

Bryan R. Loyola, Sandia National Laboratories (United States)

David Dennis Lee Mascarenas, Los Alamos National Laboratory

(United States)

Sami F. Masri, The University of Southern California (United States)

Akira Mita, Keio University (Japan)

Tomonori Nagayama, The University of Tokyo (Japan)

Yiqing Ni, The Hong Kong Polytechnic University (Hong Kong, China)

Hae Young Noh, Carnegie Mellon University (United States)

Irving J. Oppenheim, Carnegie Mellon University (United States)

Wieslaw M. Ostachowicz, The Szewalski Institute of Fluid-Flow Machinery (Poland)

Jinping Ou, Dalian University of Technology (China)

Shamim N. Pakzad, Lehigh University (United States)

Seunghee Park, Sungkyunkwan University (Korea, Republic of)

Jin-Song Pei, The University of Oklahoma (United States)

Michael K. Philen, Virginia Polytechnic Institute and State University (United States)

Paul Reynolds, University of Exeter (United Kingdom)

Massimo Ruzzene, Georgia Institute of Technology (United States)

Liming W. Salvino, Office of Naval Research Global (United States)

Jeffrey T. Scruggs, University of Michigan (United States)

Fabio Semperlotti, University of Notre Dame (United States)

Sung-Han Sim, Ulsan National Institute of Science and Technology

(Korea, Republic of)

Billie F. Spencer Jr., University of Illinois at Urbana-Champaign (United States)

Wieslaw J. Staszewski, AGH University of Science and Technology (Poland)

Lizhi Sun, University of California, Irvine (United States)

R. Andrew Swartz, Michigan Technological University (United States)

Masayoshi Tomizuka, University of California, Berkeley (United States)

Ming L. Wang, Northeastern University (United States)

Xingwei Wang, University of Massachusetts Lowell (United States)

Yang Wang, Georgia Institute of Technology (United States)

Chung-Bang Yun, Ulsan National Institute of Science and Technology

(Korea, Republic of) 
Yunfeng Zhang, University of Maryland, College Park (United States)

Li Zhou, Nanjing University of Aeronautics and Astronautics (China)

Daniele Zonta, Università degli Studi di Trento (Italy)

\section{Session Chairs}

Keynote Session

Hoon Sohn, KAIST (Korea, Republic of)

Kon-Well Wang, University of Michigan (United States)

1 Novel Approaches to Energy Harvesting

Seunghee Park, Sungkyunkwan University (Korea, Republic of)

Ying Huang, North Dakota State University (United States)

2 Vibrations and SHM of Bridge Structures

Hae Young Noh, Carnegie Mellon University (United States)

Chin-Hsiung Loh, National Taiwan University (Taiwan)

3 Spatial Sensing by Tomographic Methods

Kenneth J. Loh, University of California, Davis (United States)

Hoon Sohn, KAIST (Korea, Republic of)

$4 \quad$ Nanoengineered Thin Film Sensors I

Jerome P. Lynch, University of Michigan (United States)

Andrew R. Burton, University of Michigan (United States)

$5 \quad$ Advanced Ultrasonic Methods for SHM I

Jung-Wuk Hong, KAIST (Korea, Republic of)

Fabio Semperlotti, University of Notre Dame (United States)

6 Novel System Identification and Damage Detection Strategies

Yang Wang, Georgia Institute of Technology (United States)

Michael B. Wakin, Colorado School of Mines (United States)

7A Big Data and Data Analytics for SHM

Genda Chen, Missouri University of Science and Technology

(United States)

Donghyeon Ryu, New Mexico Institute of Mining and Technology

(United States)

7B Sensing Systems for Flight Control

Simon Laflamme, lowa State University (United States)

Wei Song, The University of Alabama (United States)

8A Actuation and Feedback Control Technologies

Benjamin Winter, Michigan Technological University (United States) 
8B Nonlinear Ultrasonics for Damage Detection

Irving J. Oppenheim, Carnegie Mellon University (United States)

Hoon Sohn, KAIST (Korea, Republic of)

9A SHM of Concrete Structures

Neil A. Hoult, Queen's University (Canada)

Hui Li, Harbin Institute of Technology (China)

9B Advances in Wireless Sensors for SHM

Jerome P. Lynch, University of Michigan (United States)

Alireza Bayat, University of Nevada, Reno (United States)

10A Nanoengineered Thin Film Sensors II

Bryan R. Loyola, Sandia National Laboratories (United States)

Jerome P. Lynch, University of Michigan (United States)

10B Advanced Ultrasonic Methods for SHM II

Wieslaw M. Ostachowicz, The Szewalski Institute of Fluid-Flow Machinery (Poland)

Francesco Lanza di Scalea, University of California, San Diego (United States)

11 A Human and Humanoid Sensing and Actuation

Genda Chen, Missouri University of Science and Technology (United States)

Donghyeon Ryu, New Mexico Institute of Mining and Technology (United States)

11B Control of Wind Turbines

Simon Laflamme, lowa State University (United States)

Wei Song, The University of Alabama (United States)

12A Fiber Optic Sensing Technology

Haiying Huang, The University of Texas at Arlington (United States)

Branko Glisic, Princeton University (United States)

12B Vision-based Sensing Technologies

Chih Chen Chang, Hong Kong University of Science and Technology (Hong Kong, China)

Daniele Zonta, Università degli Studi di Trento (Italy)

13A Applications of Smart Structure Technologies

Alessandro Cattaneo, Los Alamos National Laboratory (United States)

Hoon Sohn, KAIST (Korea, Republic of)

Chen Hao Jin, University of Connecticut (United States) 
13B Next-Generation Sensors for Smart Structures

Masahiro Kurata, Kyoto University (Japan)

Didem Ozevin, University of Illinois at Chicago (United States)

14A Advanced Composite Structural Systems

David Dennis Lee Mascarenas, Los Alamos National Laboratory (United States)

Bryan R. Loyola, Sandia National Laboratories (United States)

14B Modeling Techniques for Smart Structures

Shamim N. Pakzad, Lehigh University (United States)

Mohammad Reza Jahanshahi, Purdue University (United States) 